\title{
A Signal-on Electrochemiluminescence Immunosensor for Detecting Alpha Fetoprotein Using Gold Nanoparticle- Graphite-Like Carbon Nitride Nanocomposite as Signal Probe
}

\author{
Jiangru Wang ${ }^{1}$, Xinli Guo ${ }^{1}$, Huijuan Li $^{2}$, Yucheng Jin $^{1}$, Lusheng Chen ${ }^{1, *}$, Qi Kang ${ }^{1, *}$ \\ ${ }^{1}$ College of Chemistry, Chemical Engineering and Materials Science, Collaborative Innovation Center \\ of Functionalized Probes for Chemical Imaging in Universities of Shandong, Key Laboratory of \\ Molecular and Nano Probes, Ministry of Education, Shandong Provincial Key Laboratory of Clean \\ Production of Fine Chemicals, Shandong Normal University, Jinan 250014, P. R. China. \\ ${ }^{2}$ College of Chemical and Environmental Engineering, Shandong University of Science and \\ Technology, Qingdao, 266590, P.R.China \\ *E-mail:chls61@126.com; qikang@sdu.edu.cn
}

doi: $10.20964 / 2017.10 .75$

Received: 3 June 2017 / Accepted: 14 August 2017 / Published: 12 September 2017

The determination of cancer biomarkers plays an important role in early cancer screening and disease diagnosis. Herein, we designed a sandwiched "signal on" electrochemiluminescence (ECL) immunosensor to alpha fetoprotein (AFP) using gold nanoparticle-modified graphite-like carbon nitride nanosheets (Au NPs@g- $\mathrm{C}_{3} \mathrm{~N}_{4} \mathrm{NSs}$ ) nanocomposite as the signal tag. The capture probes of primary antibodies were immobilized on the surface of the modified electrode of MSAPANI/MWCNTs/GCE with high electrical conductivity. Where MSA, PANI, MWCTs, and GCE are mercaptosuccinic acid, polyaniline and multiwalled carbon nanotubes, and glass carbon electrode, respectively. The amount of AFP was quantified by the cathodic ECL of $\mathrm{g}_{-} \mathrm{C}_{3} \mathrm{~N}_{4} \mathrm{NSs}$ from the sandwich type immunocomplex. Under the optimized conditions, the proposed sensing strategy provides a measurable AFP concentration range from $0.1 \mathrm{pg} \mathrm{mL}^{-1}$ to $1 \mathrm{ng} \mathrm{mL}{ }^{-1}$ with a limit of detection of $0.03 \mathrm{pg} \mathrm{mL}^{-1}$. Such protocol exhibits high sensitivity, good stability and promising potential applications in clinical analysis.

Keywords: Electrochemiluminescence; immunosensor; graphite-like carbon nitride; alpha fetoprotein

\section{$\underline{\text { FULL TEXT }}$}

(C) 2017 The Authors. Published by ESG (www.electrochemsci.org). This article is an open access article distributed under the terms and conditions of the Creative Commons Attribution license (http://creativecommons.org/licenses/by/4.0/). 